\title{
Pain and treatment of carious primary teeth
}

Dental pain and dental treatment of young children attending the general dental service

\section{K. M. Milsom, M. Tickle and A. S. Blinkhorn Br Dent J 2002; 192: 280-284}

\section{Objectives}

The objective was to examine the relationship between dental pain (and its sequaelae), and the extent of restorative care provided for primary molars, amongst children who regularly attend a general dental practitioner.

\section{Methods}

A retrospective review of the clinical case notes of 677 children with caries who attended 50 general dental practitioners on a regular basis. Analyses were performed at the subject level. Logistic regression models were fitted for the dependent variables whether or not pain, a dental extraction for pain or sepsis and a course of antibiotics was recorded, after taking into account the proportion of carious teeth that were restored, the total number of carious teeth, the age caries was first recorded, gender and the clustering of the subjects within dental practices.

\section{Results}

Almost half of the children in the study (48\%) were recorded as having at least one episode of pain. Total decay experience in the primary molars was a significant predictor of pain, extraction due to pain or sepsis and prescription of antibiotics. There was no significant association between the proportion of carious teeth restored and each of the three dependent variables.

\section{Conclusions}

For those children who regularly attend their general dental practitioner and who have decay in their primary molars, dental pain is a common finding. Total decay experience in primary molars is the principal predictor of pain, extraction due to pain and the need for antibiotics, whilst the level of restorative care in the primary dentition is less important. In order to reduce the incidence of dental pain in young children, effective methods of preventing caries at the individual and public health levels need to be expanded.

\section{IN BRIEF}

- Almost half of all the study children who regularly attended GDPs in the Northwest of England experienced at least one episode of dental pain associated with their primary molars.

- Total decay experience associated with the primary molars was a significant predictor of pain.

- There was no association found between the proportion of primary molars that were restored and the presence of recorded pain.

- If restorative care is not an important factor in predicting dental pain,but total decay experience is, then prevention of the disease rather than its repair should form the focus of care for young children.

- Further studies are required to develop an evidence base in this relatively unresearched area.

\section{COMMENT}

This is an interesting and potentially controversial paper. The authors suggest that: among children attending a general dental practice on a regular basis, pain, sepsis and extractions resulting from tooth decay were a sizeable problem; and increased levels of restorative care did not lead either to reduced levels of pain, or to reduced levels of extraction therapy

No doubt some readers will argue that these findings are simply a reflection of an under-funded, under-skilled and inadequate approach to paediatric dentistry within the General Dental Service of the NHS. Others will argue that the paper reinforces the message that a restorative approach is doomed to failure from the outset, and that this should be replaced by a comprehensive primary health care approach with a strong emphasis on health promotion. Yet others will take the opportunity to castigate the present and previous governments for their perceived lack of commitment to introducing water fluoridation. If readers find themselves drawn to any of these conclusions I would suggest they re-read the paper critically.

The study involves a retrospective review of the clinical care records of a group of 677 children who attended 50 general dental practices on a regular basis over a 9 -year period. This study design poses a number of problems. First, all children included had to have experienced at least one primary molar with interproximal caries. Children with no experience of decay in their primary molar teeth who might account for more than half of the regularly attending young children in a general dental practice ${ }^{1}$ were excluded from the study. Second, case notes are often deficient, particularly in recording preventive advice and treatment. ${ }^{2}$ Indeed, a recent leader in this journal strongly reinforces that message. ${ }^{3}$ Thirdly, the extent of dental caries (measured as the number of decayed primary molars) was only weakly associated with the prevalence of pain, sepsis and extractions (the odds ratios were around 1.2). Yet this finding does not tally with the data from epidemiological studies in fluoridated areas ${ }^{4}$ where the effects on pain, sepsis and extractions were marked. Finally, teasing out cause and effect from this type of study is notoriously difficult.

However the authors are well aware of the limitations of their study. They note that 'these primary studies highlight an anomaly, and lay the foundations to move from observational research to studies designed to examine the effectiveness of restorative care within the primary care setting: The authors are to be congratulated on raising this important anomaly and they and others should be encouraged to continue this research and implement prospective studies.

\section{A Lennon}

Professor of Dental Public Health, University of Liverpool

1. O'Brien M. Children's dental health in the United Kingdom. London: OPCS, 1993

2. Lennon M A, Worthington HV, Coventry P, Mellor A C and Holloway P J. The Capitation study. 2. Does capitation encourage more prevention? Br Dent J 168: 213-215.

3. Grace M. Just for the record. Br Dent J 191: 417.

4. Duxbury J T, Lennon M A, Mitropoulos C M, Worthington HV. Differences in caries levels in 5-year-old children in Newcastle and North Manchester in 1985. BrDent」 162: $457-458$. 